\title{
SOBRE PESSOA E REFERENCIALIDADE NO
}

PORTUGUÊS

\section{On person and referenciality in Portuguese}

\author{
Danniel da Silva Carvalho*
}

\begin{abstract}
RESUMO
O objetivo deste trabalho é discutir o comportamento da $1^{\mathrm{a}}$ pessoa do singular no português a partir de dados de primeira pessoa do singular não marcada no português brasileiro (PB) encontradas em dados do português afro-brasileiro coletados na comunidade de Helvécia, Bahia e Muquém, Alagoas. 0 trabalho parte da análise de Brito e Carvalho (2014), que relacionam a estrutura de pro ao mecanismo de licenciamento que torna possível que, ainda que a morfologia de primeira pessoa do singular não esteja presente na flexão verbal, esta seja a referência recuperada por pro, uma vez que, segundo Rizzi (1986), pro deve ser identificado e, para tanto, herda os traços- $\varphi$ do licenciador. Esta análise é, então, reinterpretada a partir da noção de relativização de traços, proposta inicialmente por Preminger (2014), assumindo-se um mecanismo de concordância também relativizado.
\end{abstract}

Palavras-chave: $1^{a}$ pessoa pronominal; referencialidade; relativização de traços- $\varphi$.

\section{ABSTRACT}

The aim of this paper is to describe the pronominal structure of null pronoun pro in first person singular agreement contexts, which are not morphologically marked in Brazilian Portuguese (BP) and are found in Afro-Brazilian Portuguese data from Helvécia, Bahia and Muquém, Alagoas communities. We have tried to relate those pro structures to the licensing mechanism

* UFBA/CAPES. 
which makes the reference of pro possible even if the first person singular morphology is not present in verbal inflection, since, according to Rizzi (1986), pro must be identified and, to do so, inherits the $\varphi$-features of the licenser. To explain our data, we assume that pro must not have the same features of $\mathrm{T}$, showing a deficient structure and also there is no bond between the realization of pro and morphological verbal inflection.

Keywords: null pronouns; referentiality; $\varphi$-features.

\section{Apresentação}

Este trabalho apresenta uma revisão da proposta de Brito e Carvalho (2014), que descreve o processo de referencialidade em contextos de concordância de primeira pessoa do singular não marcada no Português Brasileiro (PB), como ilustrado em (1), estabelecendo uma comparação entre o sistema referencial de $1^{\mathrm{a}}$ pessoa da comunidade de Helvécia - BA e o da comunidade de Muquém - AL.

\section{(1) Num teve porção de fio não! ${ }^{1}$}

Ambas as comunidades foram constituídas majoritariamente por escravos (cativos ou libertos), mas estabeleceram contatos linguísticos diferenciados: Helvécia tinha uma maioria de senhores estrangeira, enquanto em Muquém conviviam, provavelmente, brancos, índios e negros nascidos em terras brasileiras. Apesar de terem a mesma idade nas datas em que as entrevistas foram coletadas, uma diferença de aproximadamente duas décadas separa o nascimento das nossas duas informantes, o que, conjuntamente às particularidades constitutivas de cada comunidade, pode determinar diferenças acentuadas nas variedades do PB adquiridas por HV-13 e F3.

Brito e Carvalho (2014) selecionaram, para análise, contextos de realização da primeira pessoa do singular na posição de sujeito, quer como pronome pleno, quer como pronome nulo, pro:

\section{HV-13 (Helvécia)}

(2) ieu gosto é de roça, num gosto de comércio não.

(3) Eu passô com minha fia no fazado mais marido dela.

(4) Fui nascido ali na roça, ficô criô lá na roça, até dobrô, casô, tudo na roça.

1 Todos os exemplos foram retirados de Brito e Carvalho (2014). 
(5) Não fuma, não bebe, só vinho, bebida minha, num fuma nada.

(6) esses bebida assim manso ieu pego, mas esse babo ieu num bebe não.

(7) eu num pode sabê, que eu tava pequeno, né?

(8) Só vejo falá nome, mas num conhece.

(9) se eu dô o senhô marcriaçon, eles me dão um côro chateado.

(10) ieu acho qu'é cas'd'idade, porque ieu mesmo num conhece não, meu senhô.

\section{F3 (Muquém)}

(11) eu nu sô da famia - mai eu entrei na famia - sô da famia - n'é - passei - passei sessenta e dois ano casada - depoi o marido morreu: fiquei n'é?

(12) Cheguei aqui cum doze ano.

(13) Eu vim de fora.

(14) e - eu posso fazê loiça não - mia fia - eu - é um sacrifício pa me levantá - eu i fazê loiça.

(15) Nunca saí - - só vô dá uma viage - pá num votá mais.

(16) - Pa cidade tem no - ôinbo - tem um ôinbo - qu'eu num sei que ôinbo que é não.

Podemos observar, nos dados de HV-13, uma oscilação na realização do pronome de primeira pessoa do singular, mesmo em contextos em que ele não pode ser sintaticamente recuperado, ou seja, com verbos que não apresentam a flexão correspondente à primeira pessoa do singular (cf. (5)). Já os dados de F3 demonstram o padrão de realização do pronome pleno na posição de sujeito, assim como de pro referencial, identificado no português brasileiro em geral, ou seja, pro como sujeito de um verbo que apresenta a morfologia de pessoa (lembrando que estamos tratando apenas da $1^{\text {a }}$ pessoa do singular).

Alguns estudos afirmam que a recuperação do referente do sujeito nulo é dada pela correferência com um DP antecedente, no caso da $3^{a}$ pessoa, ou pela presença de um morfema flexional, no caso da $1^{\text {a }}$ pessoa. Mas como explicar os dados de HV-13?

Segundo Baxter (1997, p. 281),

[i]n the Helvécia dialect, the fact that the S-V rule is variable for all person and number forms of the verb, whereas tense-aspect morphology for the present and preterite is (largely) in place, lends support to the acquisitional order tense-aspect morphemes > person number morphemes. This resembles the situation in those 
Portuguese based creoles which have, in certain cases, absorbed Portuguese preterite and imperfect morphology, but not person-number morphology.

Baxter (1997) defende, a partir de análise dos dados coletados em Helvécia, uma origem crioula para o PB, em virtude das muitas semelhanças entre fenômenos encontrados nesses dados e aqueles característicos de línguas crioulas de base lexical portuguesa. Nós não discutiremos o mérito desta questão, apenas a citamos como uma das possíveis explicações para as particularidades encontradas nos inquéritos de Helvécia.

\section{Sobre REFERENCIALIDAdE: DO ESTAdo DE ARTE AOS DADOS DO PORTUGUÊS}

Em um dos trabalhos seminais sobre pronomes nulos, Rizzi (1986) afirma que pro está sujeito às seguintes condições: a) ele deve ser licenciado, e a disponibilidade de um licenciador é determinada parametricamente; b) ele deve ser identificado; especificamente, pro herda os traços $\varphi$ do licenciador.

De acordo com Rizzi (1986, p.543), "nonarguments, quasi arguments, and referential arguments are hierarchically ordered with respect to the required richness of their content expressed in terms of $\varphi$-features". Dessa forma, "if no content is recovered, pro will be restricted to nonargumental use; if only the number specification is recovered, pro will be restricted to nonreferential uses; if both person and number are recovered, it will have the full range of uses." (RIZZI, 1986, p. 547)

Entretanto, a literatura apresenta contra-argumentos a essa proposta: a) casos complexos de línguas como o hebraico (BORER, 1983) e o irlandês (MACCLOSKEY; HALE, 1984), que admitem pro referencial em algumas pessoas e números ( $1^{\mathrm{a}}$ e $\left.2^{\mathrm{a}}\right)$, mas não em outras ( $\left.3^{\mathrm{a}}\right)$; b) línguas como o chinês (HUANG, 1989), sem um sistema de flexão verbal de pessoa e número, que licenciam o sujeito pronominal nulo, pro, correferente a um elemento nominal em uma posição que c-comanda a posição do sujeito pronominal; c) línguas crioulas em geral (ROBERTS, 1997) não apresentam sujeitos nulos referenciais.

Jaeggli e Safir (1989), em uma tentativa de incorporar as línguas pro-drop orientadas para o discurso na tipologia das línguas de sujeito nulo, formulam o Princípio da Uniformidade Morfológica ${ }^{2}$. Dessa forma, o Parâmetro do Sujeito Nulo pode ser formulado no sentido de que sujeitos

2 Um paradigma flexional $\mathrm{P}$ em uma língua $\mathrm{L}$ é morfologicamente uniforme se $\mathrm{P}$ apresenta apenas formas flexionais não derivadas (i.e., carece completamente de marcação flexional) ou apenas formas flexionais derivadas. 
nulos pode ser formulado no sentido de que sujeitos nulos são permitidos em todas e apenas nas línguas com paradigmas flexionais morfologicamente uniformes (cf. também SPEAS, 2004). são permitidos em todas e apenas nas línguas com paradigmas flexionais morfologicamente uniformes (cf. também SPEAS, 2004).

O PB contemporâneo, assim como o PAB atestado nos inquéritos de Helvécia, não se enquadram no parâmetro do sujeito nulo, assim como formulado por Rizzi (1986). No PB, o sujeito referencial nulo é enfraquecido e o paradigma de flexão verbal de pessoa e número também enfraquecido (DUARTE, 1995). Segundo Lucchesi (2009), o PB também exibe processos de variação e mudança induzidos pelo contato entre línguas, mas em um grau mais leve do que o observado nas línguas crioulas de base lexical portuguesa da África. Para o PAB, temos um algo similar ao apresentado pelo PB, mas por motivações diferentes: segundo Lucchesi (2009), as comunidades rurais afro-brasileiras foram afetadas diretamente pelo contato maciço e abrupto entre línguas na sua formação, o que impedia a realização do sujeito nulo por conta do enfraquecimento da flexão verbal influenciada por esse contato. A possibilidade de ocorrência do pro referencial surge posteriormente, a partir do contato entre essas comunidades e falantes do $\mathrm{PB}$, o que resulta em um padrão diferenciado de ocorrência do sujeito nulo, como pode ser constatado nos dados de HV-13. Já a contemporânea não realização do sujeito nulo no PB culto deve-se à reorganização que o paradigma pronominal dessa língua vem sofrendo após a inserção dos pronomes você e a gente.

Nos desenvolvimentos da Teoria de Princípios e Parâmetros, que se convencionaram chamar de Programa Minimalista (CHOMSKY, 1995 et seq.), as relações de concordância assumem outro estatuto: os traços $\varphi$ nos verbos (e nas categorias verbais) não são interpretáveis (teoria de checagem) ou valorados (teoria baseada na valoração de Agree). Eles devem, assim, ser checados/valorados pelos traços $\varphi$ do DP sujeito. pro no entanto, não tem nenhum traço valorado, sendo dependente de $\mathrm{T}$ para o estabelecimento de suas propriedades referenciais. A hipótese levantada, naquele momento, é a de que pro tem traços interpretáveis, ocupa o Spec-TP e funciona exatamente como um pronome realizado. $\mathrm{O}$ fato de pro ser silenciado é uma questão para PF [análise de deleção: sujeitos nulos = pronomes deletados].

Anteriormente à adoção desta proposta, D é assumido como o locus da referencialidade (cf. LONGOBARDI, 1994), ou seja, para um sujeito nulo ser referencial, D tem de estar presente. Alguns anos depois, estudos assumem uma estrutura interna ainda mais complexa para os elementos pronominais, e atribuem a essas diferenças estruturais o comportamento diferenciado das expressões nominais (CARDINALETTI; STARKE, 1999). Déchaine e Wiltschko (2002), por exemplo, classificam os pronomes em três tipos: pro-DPs, pro- $\varphi$ Ps e pro-NPs. 
Assim, a observação da estrutura interna das expressões nominais permite a estipulação de novos mecanismos de concordância: a) em línguas completamente pro-drop, T carrega um traço $\mathrm{D}$, e, por consequência, sujeitos nulos são sempre referenciais (ou seja, são $\varphi$ Ps que recebem a capacidade de referir a um indivíduo ou grupo de D em T); b) em línguas semi pro-drop, $\mathrm{T}$ não carrega um traço $\mathrm{D}$, e sujeitos nulos são, por consequência, interpretados como não referenciais na ausência de outro provedor de D; os sujeitos nulos referenciais são DPs e não requerem $\mathrm{D}$ de $\mathrm{T}$, i. e., $1^{\mathrm{a}} \mathrm{e} 2^{\mathrm{a}}$ pessoas são DPs, mas a $3^{a}$ pessoa é um $\varphi P$; em línguas que não permitem pro-drop, $T$ não carrega um traço $\mathrm{D}$ e requerimentos fonológicos fortes de EPP não permitem a realização de sujeitos nulos.

Por conseguinte, temos uma nova tipologia de sujeitos nulos, que podem ser: a) $\varphi$ Ps: interpretados como não referenciais na ausência de $D$ em T; b) DPs: interpretados como referenciais.

Roberts (2007) assume com Holmberg (2005) a diferença entre línguas com sujeito nulo e línguas sem sujeito nulo (i.e., presença versus

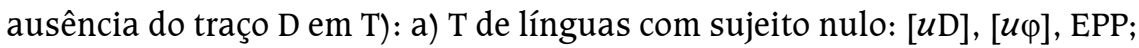
b) T de línguas sem sujeito nulo: graus variáveis de $\varphi$ empobrecido e EPP.

Assim como Holmberg, Roberts assume que sujeitos nulos são $\varphi$ Ps e que há uma conexão entre sujeitos nulos referenciais e concordância.

\subsection{LÍNGUAS DE SUJEITO NULO SEM MORFOLOGIA DE CONCORDÂNCIA}

Há línguas de pro-drop radical que permitem pro-drop apesar de carecer totalmente de morfologia de concordância verbal, como, por exemplo, algumas línguas orientais. A explicação tradicional para este fato empírico é a de que a ausência uniforme de flexão licencia pro-drop radical; a partir dos desenvolvimentos teóricos sobre a estrutura interna das expressões nominais, surge a proposta de que a estrutura morfológica de elementos pronominais em uma língua pode determinar se ela permite ou não pro-drop. Em particular, línguas com morfologia aglutinante/analítica em DPs licenciarão pro-drop por razões relacionadas aos pronomes (NEELEMAN; SZENDRóI, 2007). ${ }^{3}$ A ideia-chave é a de que o pro-drop radical é o spell-out zero de um pronome regular. Essa proposta é colocada em confronto com novos achados tipológicos, que mostram que (i) não há línguas com um paradigma pronominal flexional ou invariante que apresentem pro-drop radical; e (ii)

3 A proposta de Neeleman e Szendrói (2007) para deleção de proninais assume uma camada KP (cf. BITTNER; HALE, 1996; NEELEMAN; WEERMAN, 1999) condicionante para spell-out dessas estruturas. Entretanto, é mostrado que a primeira pessoa em PB não recorre a caso para condicionar sua realização morfológica, como será mostrado mais adiante. 
línguas que apresentam pro-drop radical devem também apresentar morfologia aglutinativa para pelo menos um traço nominal.

Como visto acima, o PB também se configura como um problema para essa classificação, uma vez que apresenta características mistas de línguas com sujeito nulo obrigatório e de línguas com sujeito pleno obrigatório. Dados de aquisição do PB (MAGALHÃES, 2006) demonstram que crianças brasileiras usam um alto percentual de sujeitos nulos de $3^{\text {a }}$ pessoa (inclusive com um referente de $1^{a}$ pessoa), o que é especialmente significativo porque vem comprovar que tem que haver algo além da morfologia flexional para garantir a manutenção desses sujeitos nulos no PB. ${ }^{4}$

\section{SOBRE CONCORDÂNCIA E RELATIVIZAÇÃo DE TRAÇOS}

Carvalho e Brito (2014) fazem uma descrição para a estrutura do pronominal nulo pro em contextos de concordância de primeira pessoa do singular não marcada no português brasileiro (PB), ilustrada nos dados em (1-16) acima. A análise se baseia na proposta de Béjar (2003), que propõe uma teoria de traços para concordância com bases minimalistas, preenchendo uma lacuna na empreitada gerativista. A análise se baseia em uma perspectiva para traços formais onde $\varphi$ codifica as propriedades nominais que tipicamente entram no processo de concordância: pessoa, número e gênero. Entretanto, tais propriedades são insuficientes para capturar exigências sintáticas de tal teoria de concordância baseada apenas em traços. Assim, a autora assume com Harley e Ritter (2002) que pronomes obedecem a uma hierarquia interna, que é responsável por sua sistematização. As tradicionais categorias pessoa e número são centrais na teoria desenvolvida por Béjar. . Segundo os autores, as relações de concordância estabelecidas pela autora são úteis à descrição de algumas relações sintáticas encontradas no PB. A abreviação tradicional de traços- $\varphi$ como um conjunto cristalizado de traços que compõem um elemento $\mathrm{R}$ (eferencial) é inviável, uma vez que não captura as relações que um elemento $R$ pode ter no sistema computacional, sendo alguns desses traços ausentes por razões independentes.

Os autores assumem que a deficiência (ausência de um dado traço na geometria), noção capiturada dos trabalhos de Cardinaletti e Starke (1999) e Harley e Ritter (2002), é responsável pela distribucionalidade do pronome.

4 Nossa intenção é descrever o licenciamento de pro nas contruções em que há uma aparente opcionalidade na escolha do spell-out pronominal, o que afasta nossa proposta de trabalhos como os de Modesto (2008), por não se tratar, como já mencionado aqui, do resultado de tópico proeminente. Para uma discussão acerca da parametrização do sujeito nulo no PB, ver Kato e Negrão (2000). 
Os reflexos de tal deficiência são sentidos sintaticamente, ou seja, têm implicações em como o mecanismo de concordância funcionará, uma vez que um pronome deficiente pode exitosamente entrar em uma relação de concordância, i.e., no caso de a derivação envolver um pronome deficiente e, ao mesmo tempo, um verbo totalmente especificado, ela convergirá, mesmo não satisfazendo certas condições para concordância (match nos termos de Chomsky (1998)), como será mostrado adiante.

De acordo com Harley e Ritter (2002), a forma de um dado pronome (e suas características) é capturada como o resultado de sua composicionalidade de traços e de suas relações sintáticas. Assumiremos, portanto, que esta composicionalidade interna é suficiente para codificar algumas características "externas", tais como referencialidade. Adicionalmente, algumas categorias externas ao nó [PARTICIPANT] (a saber, [CLASS]) serão assumidas como inter-relacionadas a ele, tornando-se este nó crucial, ao lado de [INDIVIDUATION], na variabilidade encontrada no sistema pronominal do PB.

A geometria de Harley e Ritter (2002) possibilita a subespecificação (parcial e/ou total) de um dado sistema pronominal. Sistematicamente, subespecificação codifica contraste: uma subespecificação total quando da ausência do nó [PARTICIPANT], por exemplo, resulta na terceira pessoa, enquanto subespecificação relativa pode resultar na segunda pessoa quando o nó [PARTICIPANT] tem uma interpretação default na ausência de mais nós dependentes. Consequentemente, o traço [SPEAKER] é normalmente dependente do traço [PARTICIPANT], resultando na primeira pessoa.

Béjar (2003) introduz um novo traço à geometria de Harley e Ritter (2002), a saber, $[\pi]$. [ $\pi]$ é um nó intermediário entre a raiz do elemento referencial e o nó [PARTICIPANT]. Este é também um rótulo para a categoria pessoa. Segundo a autora, $[\pi]$ é empiricamente necessário. Por exemplo, sem ele, não participantes são obrigatoriamente subespecificados exceto pelo traço [INDIVIDUATION], e, talvez, pelo traço raiz $[R]^{5}$. A introdução deste traço é também motivada pelo fato de a subespecificação de traços para terceira pessoa poder ser o ponto de variação, uma vez que, apesar de alguns pronomes de terceira pessoa serem totalmente subespecificados, há pronomes de terceira pessoa com subespecificação parcial, os quais podem atuar como interventores. Béjar (2003) afirma que a inserção do traço $[\pi]$ é necessária como uma camada representativa, já que alguns traços categoriais devem ser incluídos dentro do nó [PARTICIPANT]. 
Como Béjar (2003) aponta, em algumas línguas esta decomposição de pessoa, como proposta por Harley e Ritter (2002), não é suficiente para codificar alguns traços que são necessários para definir certos nominais (como os de COWPER; HALL (2003)). Por exemplo, [DEFINITE] e [ANIMATE] são acarretados por [PARTICIPANT] em línguas como húngaro e algonquiano, respectivamente (BÉJAR, 2003). Estas categorias, alternativamente, podem ser requeridas pelo nó [PARTICIPANT]. Portanto, $[\pi]$ rotularia os traços que podem ser capturados entre [R] e [PARTICIPANT], assumindo a representação em (17).

\section{(17) SPEAKER $>$ PARTICIPANT $>$ \{ DEICTIC $>$ DEFINITE $>$ SPECIFIC $>\ldots>\mathrm{D}=\pi\}>\mathrm{R}$}

Entretanto, sugerimos que $[\pi]$ não codifica, mas, estruturalmente, deve dominar tais traços. Isto se dá porque alguns traços assumidos por Béjar (2003) como sendo codificados por [ $\pi$ ] podem ou não estar presentes na configuração. Se eles fossem concebidos dentro de $[\pi]$, isso feriria as condições de acarretamento. Portanto, se traços como [DEFINITE] ou [SPECIFIC] estivessem localizados dentro de $[\pi]$, isto é, codificados por $[\pi]$, seria impossível ter estes traços como distintivos em um pronome arbitrário como você, em (18).

(18) Você pensa que tá fazendo a coisa certa, mas no fim você não está.

"Alguém pensa estar fazendo a coisa certa, mas no fim esse alguém não está"

"2sg pensa estar fazendo a coisa certa, mas no fim 2 sg não está”

Neste caso, o acarretamento de traços abaixo de [D] é responsável pela leitura arbitrária: na ausência de traços como [DEFINITE], você carece de uma leitura definida, que requer dêixis. Desse modo, pessoa, como uma categoria, permanece intacta enquanto traços como [SPECIFIC] ou [DEFINITE] desempenham o papel da arbitrariedade referencial. Assim, assumimos com Béjar (2003) que o nó $[\pi]$ é uma variável que codifica outros elementos que podem ser necessários para contraste de pessoa. Assim, Carvalho e Brito assumem a estrutura em (19) como sendo a possível configuração para [R]. (19) é a entrada lexical da categoria pessoa de um dado pronome que entra na derivação, engatilhando todos os mecanismos sintáticos necessários para que ele alcance PF.

\section{(19) [R [П[PARTICIPANT[SPEAKER][ADDRESSEE]]} [D[SPECIFIC[DEFINITE]]]]] 
Adicionalmente, esta estrutura é compatível com a de Déchaine e Wiltschko (2002), diferindo no que diz respeito à localização de [D]. [D] é o traço que codifica as informações nominais de um pronome, podendo ou não, teoricamente, estar presente na configuração. Uma vez presente, o pronome se comporta como um pro-DP; na notação de Déchaine e Wiltschko (2002), uma projeção extra em $\mathrm{R}$ (ou $\varphi$, na terminologia das autoras), não sendo necessário capturar tal informação. $O$ acarretamento de traços sob $[\pi]$ permite que o paradigma pronominal prediga uma forma com referência arbitrária mesmo que esta compartilhe a mesma forma de uma outra definida. A possibilidade de se ter uma pessoa arbitrária dar-se-á, portanto, da combinação do nó [PARTICIPANT] com o nó [D] subespecificado para os traços [SPECIFIC] e [DEFINITE]:

(20) Eu começo a fumar um cigarro hoje, amanhã eu experimento x. (Contexto: um agente de controle de drogas entorpecentes sendo entrevistado por um apresentador de TV)

Em (20), eu tem uma leitura arbitrária, podendo ser substituído por outra forma arbitrária:

(21) Você/a gente/alguém começa a fumar um cigarro hoje, amanhã Você/a gente/alguém experimenta $\mathrm{x}$.

Assim, a representação dos pronomes em PB de acordo com sua composicionalidade de traços gera o seguinte resultado: $[\pi]$ é responsável sozinho pela diferenciação de primeira, segunda e terceira pessoas, como esperado. Portanto, a especificação de traços relevantes para os pronomes aqui é a seguinte:

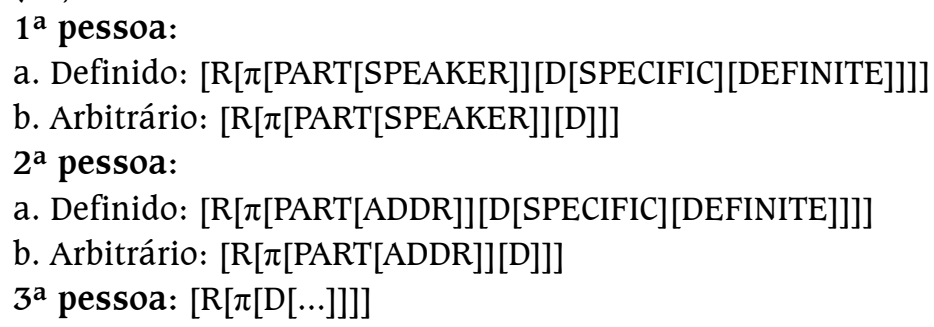

De acordo com essa proposta, um pro com referência de $1^{\text {a }}$ pessoa singular teria a mesma estrutura do pronome de $1^{\text {a }}$ pessoa do singular foneticamente realizado (eu). Portanto, em (23), extraído dos dados de H-13, teríamos a representação do pronome foneticamente nulo:

(23) Num [R[ $\pi[$ PART[SPEAKER]][D[SPECIFIC][DEFINITE] $]]$ teve porção de fio não! 
Como já fora discutido em trabalhos anteriores (BÉJAR, 2003, 2008; CARVALHO, 2008, 2011), esta decomposição de traços exige alguns ajustes no processo de concordância (Agree). A teoria de traços para lidar com Agree proposta pelos autores pode ser repensada a partir da ideia de uma concordância relativizada.

DANON (2011, p. 297) aponta que

[r] esearch on clausal syntax usually considers the internal structure of DPs to be irrelevant to the analysis of operations that take place at the clause level; and researches on the internal structure of DPs usually does not involve considerations of processes that take place above the DP level.

Essa dissociação apontada por Danon, a nosso ver, resulta na discrepância das análises dos diferentes tipos de sentenças e seus mecanismos de concordância. É sabido que é o DP quem carrega, e consequentemente, valora, os traços de concordância na sentença (cf. CHOMSKY, 1995 e seguintes). Portanto, estabelecer a própria sintaxe do DP torna-se essencial para se estabelecer a sintaxe da sentença. Isso parece óbvio, mas, como apontado por Danon, geralmente é negligenciado. Para reavaliar essa dissociação, apresentaremos a seguir a proposta de Preminger (2014), baseado em Béjar (2003) e Béjar e Rezac (2003), para restabelecer a necessária simetria entre DP e sentença.

Preminger (2014), analisando a natureza obrigatória da concordância nas línguas, discute o padrão de concordância das construções Agente-foco em Kichean, língua da família Maia. As línguas dessa família não apresentam casual em seus NPs e exibem uma estrutura ergativa-absolutiva (PREMINGER, 2014, p. 16):

\section{(24) Transitivo}

a. rat $\mathrm{x}$ - $\varnothing$-aw-ax-aj você COM-3SG.ABS.2SG.ERG-ouvir-ACT ri achim "Você ouviu o homem" o homem

b. ri achim x-a-r-ax-aj rat o homem COM-2SG.ABC-3SG.ERG-ouvir-ACT você "O homem ouviu você"

(25) Intransitivo

a. ri achim $\quad \mathrm{x}-\varnothing$-uk'lum

o homem COM-3SG.ABS-chegar

"O homem chegou" 


\section{b. rat $\quad \mathrm{x}$-at-uk'lum \\ você(SG) COM-2SG.ABS-chegar \\ "Você chegou"}

De acordo com Preminger, nos exemplos acima, o único argumento do verbo intransitivo (inacusativo) uk'lum (chegar) engatilha a mesma marca de concordância no verbo que o objeto do verbo transitivo ax (ouvir): nula $(\varnothing)$ para argumentos de terceira pessoa do singular e $a(t)$ para os de segunda pessoa do singular. Contrastivamente, o sujeito de verbos transitivos engatilha uma marcação diferente: $r(u) / u$ - para argumentos de terceira pessoa do singular e $a(w)$ para os de segunda pessoa do singular. Desta forma, o autor se propõe a investigar os padrões de concordância apresentados em construções de Agente-foco, que, de acordo com o contraste abaixo, apresenta padrão de concordância diferente dos transitivos:

(26)
a. ja rat $\mathrm{x}$-at $/{ }^{*} \varnothing$-ax-an
ri achim
FOC você COM-2SG/*3SG.ABS-ouvir- $\underline{\mathrm{AF}}$
o homem
"Foi você que ouviu o homem"
b. ja ri achim $x$-at $/ * \varnothing$-ax-an
rat
FOC o homem COM-2SG/*3SG.ABS-ouvir- $\underline{\mathrm{AF}}$ você
"Foi o homem que ouviu você"

Em (26a), o agente é de segunda pessoa e o paciente de terceira. Em (26b), o agente é de terceira pessoa e o paciente de segunda. Entretanto, a morfologia do verbo permanece a mesma em ambas as construções. Contra a hipótese de que as estruturas absolutivas em Kichean possuem dois morfemas de concordância, um para a terceira pessoa do singular e outra para a segunda pessoa do singular, o autor argumenta que há uma provável hierarquia de pessoa nas relações de concordância nesse tipo de construção, e que essas relações não podem ser vistas de forma simplista como uma relação verbo-sujeito ou verbo-objeto. Para ilustrar essa hipótese, Preminger mostra que da mesma forma que a relação $2^{\mathrm{a}} / 3^{\mathrm{a}}$ pessoas, a primeira pessoa tem precedência sobre a terceira no engatilhamento de concordância:
a. ja yïn $\mathrm{x}$-in/*ø-ax-an
ri achim FOC me COM-1SG/*3SG.ABS-ouvir- $\underline{\mathrm{AF}}$
o homem "fui eu que ouvi o homem"
b. ja ri achim $x$-in/* $\varnothing$-ax-an yïn FOc o homem COm-1SG/*3SG.ABS-ouvir- $\underline{\mathrm{AF}}$ me "foi o homem que me ouviu" 
Ainda, além das relações de pessoa, número parece também interferir nas relações de concordância para a terceira pessoa, uma vez que o plural tem precedência ao singular:

(28)

a. ja rje' $x$-e $/{ }^{*} \varnothing$-tz'et-ö rja' FOC eles COM-3-PL/*3SG.ABS-ver-AF ele "foram eles que viram ele"

b. ja rja $\mathrm{X}$-e ${ }^{*} \varnothing$-tz'et-ö rje' FOC ele COM-3PL/*3SG.ABS-ver-AF eles "foi ele que viu eles"

Finalmente, o autor aponta que argumentos de $1^{\mathrm{a}} / 2^{\mathrm{a}}$ pessoas tem precedência sobre a terceira independentemente de número:

a. ja rje' $x$-i/*oj-tz'et-ö rje' FOC eles COM-1SG/*1PL.ABS.ver--AF me "foram eles que me viram"

b. ja yïn $\mathrm{x}$-i/*oj-tz'et-ö rje' FOC me COM1SG./*1PL.ABS.ver-AF eles "Fui eu que viu eles"

(30)

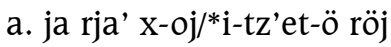
FOC ele COM-1PL/*1SG.ABS.ver-- $\underline{\mathrm{AF}}$ nos "foi ele que nos viu"

b. ja röj x-oj/*i-tz'et-ö rja' FOC nos COM-1PL/*1SG.ABS.ver--AF ele "fomos nós que vimos ele"

Podemos observar nos exemplos acima que a concordância se dá sempre com a $1^{\text {a }}$ pessoa, independentemente do número da $3^{\mathrm{a}}$. $O$ autor afirma que o mesmo ocorre com a $2^{\mathrm{a}}$ pessoa, apesar de não apresentar dados, e aponta a impossibilidade da combinação de $1^{\mathrm{a}}$ e $2^{\mathrm{a}}$ pessoas nesse tipo de estrutura. ${ }^{6}$ Assim, Premiger propõe o paradigma de concordância abaixo para as construções agente-foco em Kichean: 
(31)

Full agreement paradigm for Kichean AF (PREMINGER, 2014, p. 21)

\begin{tabular}{cc}
$\begin{array}{c}\text { SUBJ, OB }\} \\
\Phi \text {-features }\end{array}$ & $\begin{array}{r}\text { Agreement morphology } \\
\text { on the AF verb }\end{array}$ \\
\hline$\{3 \mathrm{sg}, 3 \mathrm{sg}\}$ & $3 \mathrm{sg}$ \\
$\{3 \mathrm{pl}, 3 \mathrm{sg}\}$ & $3 \mathrm{pl}$ \\
$\{3 \mathrm{pl}, 3 \mathrm{pl}\}$ & $3 \mathrm{pl}$ \\
\hline$\{1 \mathrm{sg}, 3 \mathrm{sg}\}$ & $1 \mathrm{sg}$ \\
$\{2 \mathrm{sg}, 3 \mathrm{sg}\}$ & $2 \mathrm{sg}$ \\
\hline$\{1 \mathrm{pl}, 3 \mathrm{sg}\}$ & $1 \mathrm{pl}$ \\
$\{2 \mathrm{pl}, 3 \mathrm{sg}\}$ & $2 \mathrm{pl}$ \\
\hline$\{1 \mathrm{sg}, 3 \mathrm{pl}\}$ & $1 \mathrm{sg}$ \\
$\{2 \mathrm{sg}, 3 \mathrm{Pl}\}$ & $2 \mathrm{sg}$ \\
\hline$\{1 \mathrm{pl}, 3 \mathrm{sg}\}$ & $1 \mathrm{pl}$ \\
$\{2 \mathrm{pl}, 3 \mathrm{pl}\}$ & $2 \mathrm{pl}$
\end{tabular}

a. In the notation $\{\varphi 1, \varphi 2\}$, which of the $\Phi$-feature specification belongs to the subject and which to the object is fully commutative.

b. Combinations not listed here are ruled out either on binding-theoretical grounds (e.g., $\{1 \mathrm{sg}, 1 \mathrm{pl}\})$ or because of the AF person restriction $[\ldots]$.

Para resolver o problema, Preminger adota uma proposta derivacional para concordância- $\varphi$. A proposta se baseia na Restrição Pessoa-Caso de Béjar e Rezac (2003), uma restrição para a distribuição dos traços de pessoa entre múltiplos argumentos internos de um mesmo predicado. Preminger captura da proposta de Béjar e Rezac uma de suas concepções nucleares: ideia de que as sondas sintáticas para os traços de pessoa e para os traços de número são distintas. A ideia de relativização das sondas dos traços- $\varphi$ também é discutida em Béjar (2003). Assim, para o Kichean, o autor verifica que as sondas de pessoa e número nesta língua são relativizadas para olhar apenas para os membros marcados de suas respectivas geometrias de traços ([participant] e [plural], respectivamente) ${ }^{7}$ Vamos nos restringir à discussão sobre esses traços- $\varphi$ apenas, deixando o caso de lado devido ao escopo deste trabalho e à irrelevância deste traço para nossa proposta.

A principal contribuição para o presente trabalho, capturada da análise de Preminger para as construções agente-foco no Kichean, é a pos-

7 Veremos adiante a proposta de HARLEY e RITTER (2002), em que se baseia essa geometria de traços.

Revista Letras, Curitiba, N. 91 P. 131-157, JAN./JUN. 2015.

ISSN 2236-0999 (VERSÃo ELETRÔNICA) 
sibilidade de lidar com pares sonda-alvo aparentemente diferentes: as construções Agente-foco do Kichean envolvem uma única marca de concordância e dois argumentos não oblíquos e a escolha de qual argumento estabelecerá concordância com o predicado depende de restrições estruturais desses argumentos. Grosso modo, a concordância dessas construções no Kichean seguem a seguinte hierarquia:

$$
1^{\mathrm{a}} / 2^{\mathrm{a}} \text { pessoa/ } 3^{\mathrm{a}} \text { pessoa } \mathrm{plural} / 3^{\mathrm{a}} \text { pessoa singular }
$$

Para ilustrar que o fenômeno apresenta um princípio universal para o tratamento de traços formais, o autor mostra que o que parece acontecer em Kichean é o que ocorre interrogativas deslocadas em uma língua como o inglês. A partir do que Nervins (2011) chama de omnivorous agreement, que seria a habilidade dessas estruturas de "saltar" o sujeito se ele não apresentar os traços "certos", indo em direção do objeto, o autor sugere que o que ocorre nas estruturas agente-foco em Kichean é o mesmo que ocorre com o traço [wh], que ignora os possíveis alvos uma vez que eles não possuem compatibilidade.

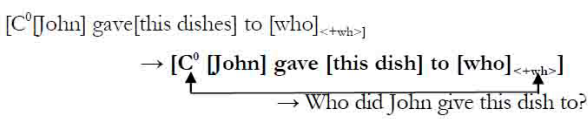

Em (33), há pelo menos três $\mathrm{X}^{0}$ que são potenciais alvos para a sonda, e entre esses alvos, alguns são mais próximos. Crucialmente, a sonda é capaz de ignorar esses alvos uma vez que eles não possuem o traço que ela procura - [wh]. Preminger lembra que esta é a porção "relativizada" da Minimalidade Relativizada (cf. RIZZI, 1990, entre outros). Analogamente, o que acontece nos exemplos em (16) é o mesmo que acontece em (33): o padrão de concordância de (16), da mesma forma que o padrão da interrogativa $\mathrm{C}^{\circ}$, envolve uma sonda que procura uma alvo XP que possua um traço particular ([wh] no caso da interrogativa $\mathrm{C}^{0}$, [plural] e/ou [participant] no caso das construções Agente-foco em Kichean). As sentenças em (16) seriam analisadas como em (34): 


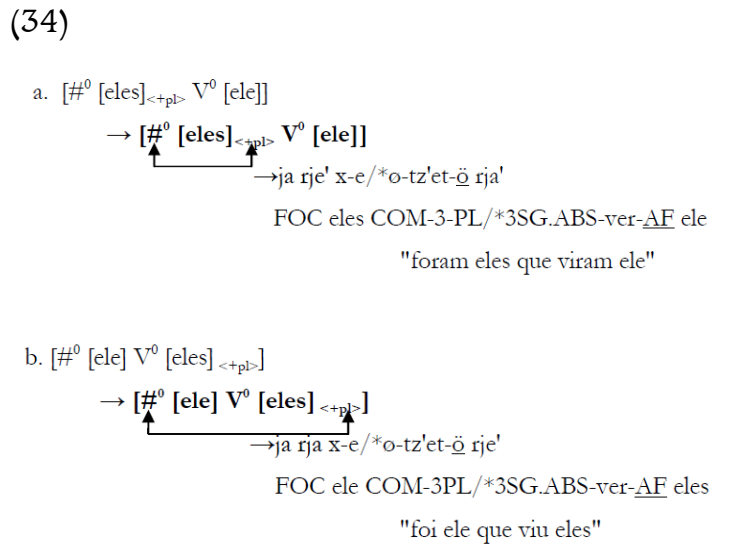

Para tal análise, Preminger lança mão de uma geometria de traços, inicialmente proposta por Harley e Ritter (2002) e adaptada por Béjar (2003) para lidar com o fenômeno dos intervenientes. Segundo Preminger (2014, p. 45), "[a] feature geometry specifies the internal structure of the space of possible feature values, encoding implicatorial relations between the occurrence of different values." Assim, o autor adota a seguinte composição para os traços- $\varphi$ :

A simplified $\varphi$-feature geometry

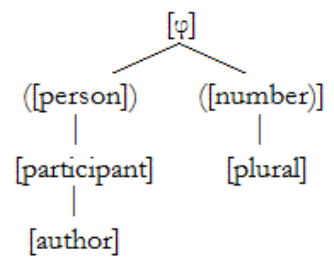

De acordo com essa geometria, o traço [participant] é o que distingue os pronomes de $1^{\mathrm{a}} / 2^{\mathrm{a}}$ pessoa dos de $3^{\mathrm{a}}$ e outros NPs; o traço [author] distingue $1^{\mathrm{a}}$ e $2^{\mathrm{a}}$ pessoas; e [plural], singular e plural. Por razões de acarretamento (cf. BÉJAR, 2003; CARVALHO, 2008), a presença de [author] pressupõe a presença de [participant], excluindo a possibilidade de se gerar algo como "autor não participante". Nessa proposta, o traço [ $\varphi]$ é um nó compartilhado por todos os nominais. De acordo com Preminger (2014, p. 46), "[i]f there is such thing as a "D-feature", which defines the natural class of extended nominal projections, and which must be visible on the 
maximal extended projection for a nominal to count as nonoblique, then this might be that feature [...]."

Ainda, o autor assume que uma geometria de traços- $\varphi$ não é relevante apenas para a realização morfológica dos pronominais, mas também é crucial na sintaxe. Para o autor, tal proposta responde à questão de como uma sonda "pula" um potencial alvo, como nas estruturas agente-foco em Kichean. A noção de subconjunto/superconjunto capturados nos trabalhos de Béjar por Preminger pode ser aplicada na proposta de valoração dos traços, como ilustrado abaixo.

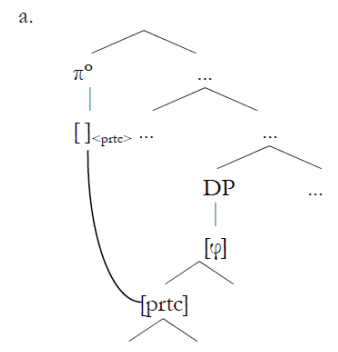

b.

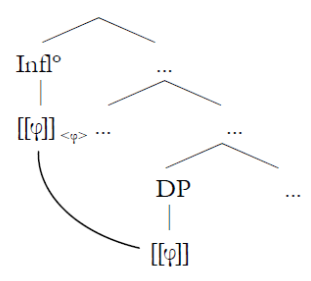

(37) feature-geometric valuation: Kichean $\pi^{o}$

a.

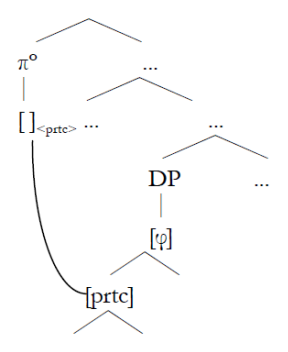


b.

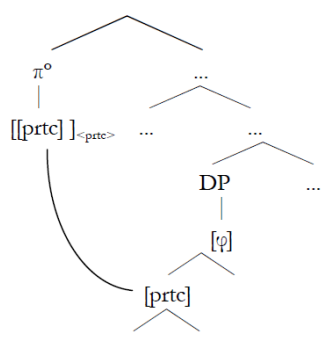

(38) feature-geometric valuation: Kichean \#o

a.

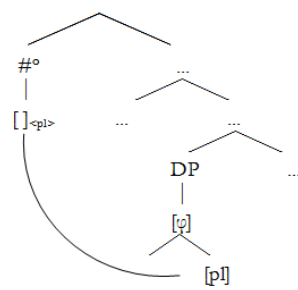

b.

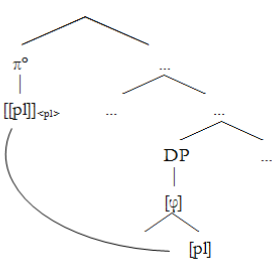

O autor finaliza a análise apontando o fato de que esse mecanismo de valoração não altera as questões apresentadas em relação à obrigatoriedade de uma concordância- $\varphi$. Lembra ainda que a proposta de traços não-interpretáveis de Chomsky $(2000,2001)$ pode ser facilmente adaptada ao modelo apresentado acima. A essência de uma proposta de geometria de traços consiste na possibilidade de unificação dos padrões de que traços podem ou não poder ser "ignorados" por uma sonda- $\varphi$. Uma interpretação desse padrão pode, a meu ver, oferecer uma análise unificada da referência de pessoa no português. 


\section{REFERÊNCIA E RELATIVIZAÇÃO DE TRAÇOS NO PORTUGUÊS}

Com base no que foi exposto acima, assumirei com Preminger que uma concordância relativizada é o que permite referência de $1^{a}$ pessoa nos dados de (2-16). Desta forma, a análise em (36-38) é o que explica tal fenômeno.

A impossibilidade de valoração deste traço é o que acarreta a aparente "falha de concordância", o que não impede Agree de obter sucesso. Isso se dá pelo fato de haver um nó responsável pela representação de qualquer elemento referencial independentemente. Esse nó é chamado de traço $[\pi]$ por Carvalho (2008) e domina os demais traços [ $\pi$ ]. Essa generalização é capturada a partir das própria motivação de Agree.

Proponho, então, que a estrutura de um [ $[$ ] é como (39):

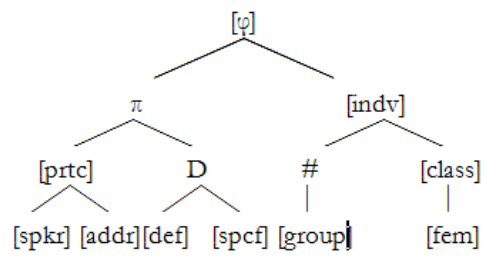

Assumiremos a proposta de Preminger (2014) para representação de $[\varphi]$ com as alterações sugeridas para a composição de traços para o PB em Carvalho (2008). [ $\varphi$ ] é o nó raiz da estrutura de concordância- $\varphi$. Sua total subespecificação gera estruturas maximamente subespecificadas, i.e., que podem ocorrer em qualquer posição sintática, seja ela A ou Ā. Os traços da notação em (39) que são relevantes para nossa discussão são descritos abaixo: ${ }^{8}$

8 Na notação de Harley e Ritter (2002), outros traços são dominados pelo nó [class]. Entretanto, esses traços não são relevante para a sintaxe dos nomes em português brasileiro, como apontado por Carvalho (2008). Mesmo o traço [fem] parece não ser para a sintaxe se adotarmos uma teoria cuja concordância só é licenciada para fins de merge (interno ou não), o que não parece ser o caso de gênero. Nas construções que envolvem concordância de gênero no português, tais estruturas parecem ter fins apenas morfofonológicos, autorizando ou não a presença de morfemas de gênero em PF. 
Quadro 1 - Notação de traços relevantes para valoração de pessoa e número

\begin{tabular}{|c|c|}
\hline Traço & Notação \\
\hline$[\pi]$ & $\begin{array}{l}\text { representa a categoria pessoa. A presença deste traço corresponde ao } \\
\text { requerimento de pessoa como categoria dominante em uma hierarquia } \\
\text { para } \varphi \text { (cf. NOYER, 1992) }\end{array}$ \\
\hline [D] & $\begin{array}{l}\text { este traço corresponde ao traço [N] (CHOMSKY, 1965) e caracteriza } \\
\text { nominais. A presença deste traço identifica o nominal como sendo um } \\
\text { argumento, como defendem alguns autores. Este traço também domina } \\
\text { a projeção que compreende traços como [DEFINITE] e [SPECIFIC] }\end{array}$ \\
\hline [participant] & $\begin{array}{l}\text { este traço caracteriza os participantes do processo discursivo }\left(1^{\mathrm{a}} \text { e } 2^{\mathrm{a}}\right. \\
\text { pessoas). A ausência deste traço e, consequentemente, dos traços por } \\
\text { ele dominados, caracteriza a } 3^{\mathrm{a}} \text { pessoa, nos termos de Benveniste, a } \\
\text { não-pessoa }\end{array}$ \\
\hline [speaker] & $\begin{array}{l}\text { a presença do traço [SPEAKER] imediatamente dominado pelo traço } \\
\text { [PARTICIPANT] define o pronome como sendo o falante no processo } \\
\left.\text { discursivo ( } 1^{\text {a }} \text { pessoa }\right) \text {. }\end{array}$ \\
\hline [addressee] & $\begin{array}{l}\text { este traço caracteriza o ouvinte no processo discursivo. A possibilidade } \\
\text { de se ter uma } 2^{\text {a }} \text { pessoa arbitrária segue as mesmas condições aponta- } \\
\text { das para uma } 1^{\text {a }} \text { pessoa arbitrária }\end{array}$ \\
\hline [specf] & $\begin{array}{l}\text { denota um indivíduo particular (ou grupo de indivíduos). Um DP que } \\
\text { apresenta o traço [SPECIFIC] é interpretado como "um indivíduo que é } \\
\text { conhecido pelo falante" }\end{array}$ \\
\hline [def] & $\begin{array}{l}\text { o traço [DEFINITE] define quando um DP se refere a algo presente no } \\
\text { universo discursivo }\end{array}$ \\
\hline [indv] & $\begin{array}{l}\text { representa a distribuição de entidades no mundo de acordo com suas } \\
\text { propriedades discursivas independentes, tais como classe e quantifi- } \\
\text { cação }\end{array}$ \\
\hline [\#] & $\begin{array}{l}\text { a presença deste traço determina a quantificação do nominal. [\#] sozi- } \\
\text { nho caracteriza uma leitura singular do nominal }\end{array}$ \\
\hline
\end{tabular}

Voltando ao fenômeno discutido neste trabalho, pronomes foneticamente nulos teriam, na sintaxe, as mesmas propriedades dos demais pronomes, uma vez que o material fonético estará disponível apenas após a sintaxe. Assim, um pro com referência de $1^{\text {a }}$ pessoa singular teria a mesma estrutura do pronome de $1^{\text {a }}$ pessoa do singular foneticamente realizado $(e u)$.

Dessa forma, dados como os apresentados abaixo em (40) tem como estrutura de traços dos pares sonda-alvo as apresentadas nas tabelas abaixo. Estes dados representam os padrões de realização da primeira pessoa do singular nos dados: pronome foneticamente realizado de $1^{\text {a }}$ pessoa do singular com verbo morfologicamente realizado na $1^{\text {a }}$ pessoa (40a); pronome 
foneticamente não realizado de $1^{\text {a }}$ pessoa do singular com verbo morfologicamente realizado na $1^{\text {a }}$ pessoa $(40 \mathrm{~b})$; pronome foneticamente realizado de $1^{\text {a }}$ pessoa do singular com verbo morfologicamente realizado na $3^{\text {a }}$ pessoa (40c); pronome foneticamente não realizado de $1^{\mathrm{a}}$ pessoa do singular com verbo morfologicamente realizado na $3^{\text {a }}$ pessoa (40d).
(40)
a. Eu sei não: mia fia
b. Cheguei aqui cum doze ano
c. Eu passô com minha fia no fazado mais marido dela.
d. Num teve porção de fio não

Tabela 1 - Agree dos traços de pessoa com pares sonda-alvo (40a)

\begin{tabular}{ll}
\hline Sonda (sei) & Alvo $(e u)$ \\
\hline & {$[\pi]$} \\
& {$[$ participant] } \\
& {$[$ speaker] } \\
& \\
\hline$[u \pi]$ & Agree é bem sucedido \\
{$[$ uparticipant] } & \\
{$[$ uspeaker] } & \\
\hline
\end{tabular}

Tabela 2 - Agree dos traços de pessoa com pares sonda-alvo (40b)

\begin{tabular}{ll} 
Sonda(cheguei) & Alvo $(-)$ \\
\hline & {$[\pi]$} \\
& {$[$ participant $]$} \\
& {$[$ speaker $]$} \\
& \\
\hline$[u \pi]$ & Agree é bem sucedido \\
{$[u$ participant $]$} & \\
{$[u$ speaker $]$} &
\end{tabular}


Tabela 3 - Agree dos traços de pessoa com pares sonda-alvo (40c)

\begin{tabular}{ll} 
Sonda (passô) & Alvo $(\mathrm{eu})$ \\
\hline & {$[\pi]$} \\
& {$[$ participant $]$} \\
& {$[$ speaker] } \\
\hline$[u \pi]$ & Agree é bem sucedido \\
\hline
\end{tabular}

Tabela 4 - Agree dos traços de pessoa com pares sonda-alvo (40d)

\begin{tabular}{ll}
\hline Sonda (teve) & Alvo $(-)$ \\
\hline & {$[\pi]$} \\
& {$[$ participant $]$} \\
& {$[$ speaker $]$} \\
\hline$[\mathrm{u} \pi]$ & Agree é bem sucedido \\
\hline
\end{tabular}

Como já mencionado acima, a diferença entre os pronomes pessoais que são foneticamente realizados e os que não o são vai depender da disponibilidade dos itens morfológicos disponíveis pós-sintaticamente, com base em condições como o Elsewhere Principle. Adotaremos uma reiterpretação do Elsewhere Principle (KIPARSKY, 1973) para estabelecer a opcionalidade spell-out da primeira pessoa nos dados acima. O Elsewhere Principle pode ser formulado da seguinte maneira (NEELEMAN; SZENDRÓI, 2007, p. 685):

\section{Elsewhere Principle}

R1 e R2 são regras em competição que têm D1 e D2 como seus respectivos domínios de aplicação. Se D1 é um subconjunto de D2, então R1 bloqueia a realização de R2 em D1.

Entretanto, os autores apontam as seguintes consequências do elsewhere principle:

i. Ceteris paribus, a realização fonológica de uma categoria C terá prioridade sobre a realização de uma categoria contida em C;

ii. Ceteris paribus, a realização fonológica de uma categoria C contendo mais traços de $\mathrm{C}$ terá prioridade sobre uma realização fonológica contendo menos traços;

iii. Opcionalidade resulta se a realização fonológica de uma categoria $\mathrm{C}$ realizer menos traços de $\mathrm{C}$ do que a realização fonológica de uma categoria contendo $\mathrm{C}$. 
Assim, ainda de acordo com Neeleman e Szendrói (2007), a validade de (i) pode ser demonstrada com os verbos irregulares do inglês, cuja forma irregular (went - ir.pret) bloqueia a forma regular de go-ed (ir + particula de pretérito no inglês), uma vez que realiza uma categoria de nível mais alto e, consequentemente, inserida via uma regra de spell-out mais específica. Os efeitos de (ii) podem ser observados na maioria dos paradigmas flexionais. Os autores dão o exemplo da concordância de segunda pessoa do singular no alemão, que pode tanto ser realizada pela marca default de terceira pessoa quanto pela de primeira pessoa do singular (HARLEY; RITTER, 2002). Assim, o fato de a segunda pessoa do singular ser usada revela a preferência por um spell-out com um maior número de traços. Por fim, (iii), nas palavras de Neeleman e Szendrói (2007, p. 687), tem a seguinte consequência:

the two implications of the elsewhere principle discussed above hold [Ceteris paribus]. This is particularly important when we consider situations in which a spellout rule for a category C realizes fewer features than the spell-out rules for the categories contained in C (say A and B). In situations of this type, the elsewhere principle does not favour one realization of [C A B] over the other. A realization of $\mathrm{C}$ as $/ \mathrm{c} / \mathrm{is}$ more specific in that it targets a higher category, while a realization of $\mathrm{C}$ as /a-b/is more specific in that it spells out more features. The consequence of this stalemate is that neither form will block the other, and hence that both realizations of $\mathrm{C}$ are allowed.

Portanto, a opcionalidade do spell-out da primeira pessoa nos dados do Português Brasileiro nas variedades discutidas neste trabalho é resultado da variação prevista na gramática, condicionada pelo próprio $E l$ sewhere Principle, uma vez que a especificidades na esturura dos pronomes não implica a violação de tal princípio. ${ }^{9}$

9 Nossa proposta reforça de todo modo a Conjectura Borer-Chomsky (BAKER, 2008), que prevê que o locus de variação nas línguas naturais é restringido por propriedades traçuais dos intens lexicais (RICHARDS, 2008, p. 135):

(i) Borer-Chomsky Conjecture (BCC; 'lexical parameters')

a. "Parametric variation is restricted to the lexicon, and insofar as syntactic computation is concerned, to a narrow category of morphological properties, primarily inflectional." (Chomsky 2001: 2)

b. "The availability of variation [is restricted] to the possibilities which are offered by one single component: the inflectional component." (Borer 1984: 3) 


\section{Algumas palavras finais}

Retomando a discussão acerca do contato, com base na análise apresentada acima, podemos assumir que diferentes gramáticas podem gerar diferentes spell-outs fonéticos, mas a estrutura gramatical destas diferentes variedades do português se mantém. Nosso argumento parte do princípio de que a gramática estabelecida em contextos de contato linguístico ou não, nessas variedades, oferece um único padrão de concordância, cuja variação se dá em termos de subespecificação dos traços dos elementos participantes da operação Agree.

Nossa intenção foi apresentar uma análise unificada para os diferentes padrões morfológicos apresentados nos dados (e por que não os padrões do português em geral), reunindo as diferentes tipologias apresentadas previamente no trabalho. Assim, línguas como o chinês, por exemplo, que apresentaria problemas para as análises prévias, seriam explicadas a partir dos padrões de concordância aqui apresentados.

Ribeiro (2010), ao estudar outro inquérito de Helvécia, o da informante HV-19, em comparação aos dados de escrita de Gregório, brasileiro afrodescendente também do século XIX, mas letrado e inserido no contexto da cidade de Salvador, constata que os dados desses informantes podem ser considerados como evidências indiretas para a coexistência, no português brasileiro popular do século XIX, de ao menos duas gramáticas de sujeito nulo, ou seja, duas possibilidades de licenciamento do sujeito nulo: uma do tipo chinês, em que não há manifestação fonológica de concordância verbal (I-D), situação típica do processo de transmissão linguística irregular ou de aquisição imperfeita (LUCCHESI, 2009; ROBERTS, 2007), como, por exemplo, aquela que podemos encontrar nos inquéritos de HV-19 e HV-13; outra do tipo românico, em que a realização dos traços- $\varphi$ de V é sistemática (I+D). A hipótese defendida por Ribeiro (2008) é a de que a competição entre essas duas gramáticas deve estar presente no PB desde os seus primórdios, dadas as diferentes e diversas situações de aquisição do PB, hipótese esta que encontra consonância na análise empreendida no presente trabalho, ainda que os mecanismos utilizados para explicação dos fenômenos sejam diferenciados nas duas propostas.

Constatamos, assim, diferenças nos padrões de concordância nas gramáticas de F3 e HV-13: em F3 encontramos, quanto à realização do pro referencial de $1^{\mathrm{a}}$ pessoa, uma gramática muito semelhante àquela encontrada no PB contemporâneo. Ribeiro (2008) afirma que

falar de um enfraquecimento da morfologia verbal só seria adequado para uma parcela da população, como a representada por Gregório e as retratadas em estudos diacrônicos com base na norma 
culta; para a população representada por HV-19, simplesmente não há morfologia verbal, exceto para a $1^{\text {a }}$ pessoa do singular (embora variável) (idem, p. 96).

Com a possível conexão entre morfologia flexional e ocorrência do pro referencial, podemos supor, então, que as influências sofridas pela gramática de $\mathrm{F} 3$ tenham sido semelhantes àquelas sofridas pela gramática de Gregório (excetuando-se o letramento), ou seja, talvez a gramática de F3 já apresente influências de um contato com o PB culto - talvez aquele utilizado pelos senhores de escravos dos seus pais e avós. Já HV-13, por ser de Helvécia e sua contemporânea, apresenta, em sua gramática, um comportamento do pro referencial semelhante ao de HV-19, com todas as especificidades discutidas ao longo deste trabalho.

\section{REFERÊNCIAS}

BAKER, M. The Syntax of Agreement and Concord. Cambridge: Cambridge University Press, 2008.

BAXTER, A. Creole-like Features in the Verb System of an Afro-Brazilian Variety of Portuguese. In: SPEAS, A. K.; WINFORD, D. (Eds.). The Structure and Status of Pidgins and Creoles. Amsterdam/Philadelphia: John Benjamins, 1997. p. 265-288.

BÉJAR, S. Phi-syntax: a theory of agreement. Tese (Doutorado) - University of Toronto, 2003.

Conditions on Phi-Agree. In: HARBOUR, D; ADGER, D.; BEJAR, S. (Eds.) Phi-Theory: Phi-Features Across Modules and Interfaces. New York: Oxford University Press, 2008.

BÉJAR, S; REZAC, M. Person licensing and the derivation of PCC effect. In: PÉREZ-LEROUX, A.T.; ROBERGE, Y. (Eds). Romance linguistics: theory and acquisition. Amsterdam: John Benjamins, 2003. p. 49-62.

BORER, H. Parametric Syntax. Dordrecht: Foris, 1983.

BRITO, D. ; CARVALHO, D. Pronomes nulos e referência no português afro-brasileiro. Veredas, V. 18 , n. 1 , p. $23-43,2014$

CARDINALETTI, A; M. STARKE. The typology of structural deficiency. In: VAN RIEMSDIJK, H. (Ed.). Clitics and other functional categories in European languages. Berlin: Mouton de Gruyter, 1999. p. 145-233.

CARVALHO, D.S. A estrutura interna dos pronomes pessoais em português brasileiro. Tese (Doutorado) - Univesidade Federal de Alagoas, 2008.

CHOMSKY, N. Minimalism Inquiries: the Framework. MITWPL 15. Cambridge, MA: Mitwpl, 1998. . The Minimalist Program. Cambridge, MA: MIT Press, 1995. . Aspects of the Theory of Syntax. Cambridge, MA.: MIT Press, 1965. 
COWPER, E.; HALL, D. C. The syntactic manifestation of nominal feature geometry. In: ANNUAL CONFERENCE OF THE CANADIAN LINGUISTIC ASSOCIATION, 2002, Montréal. Proceedings... Montréal: Cahiers Linguistiques de l'UQAM, 2003. p. 55-66.

DÉCHAINE, R-M.; WILTSCHKO, M. Decomposing Pronouns. Linguistic Inquiry, v.33, n.3, p. 409-442, 2002.

DUARTE, M. E. L. A perda do princípio 'Evite Pronome' no português brasileiro. Tese (Doutorado) - Universidade Estadual de Campinas, 1995.

HARLEY, H.; RITTER, E. Person and number in pronouns: a feature-geometric analysis. Language, v. 78, p. 482-526, 2002.

HOLMBERG, A. Is there a little pro? Evidence from Finnish. Linguistic Inquiry, v. 36, n.4, p. 533-564, 2005.

HUANG, C-T. J. On the distribution and reference of empty pronouns. Linguistic Inquiry, v. 12, p. 531-574, 1984.

parameter. Dordrecht: Kluwer, 1989.

JAEGGLI, O.; SAFIR, K. (Eds.) The null subject parameter. Dordrecht: Kluwer, 1989.

KATO, M. A.; NEGRÃO, E. V. (Eds.) Brazilian Portuguese and the Null Subject Parameter. Vervuert - Iberoamericana, 2000.

KIPARSKY, P. 'Elsewhere' in phonology. In: ANDERSON, S. R.; KIPARSKY, P. (Eds.) A Festschrift for Morris Halle. Holt; Reinhart; Winston, New York: 1973. p. 93-106.

LONGOBARDI, G. Reference and proper names. Linguistic Inquiry, v. 25, p. 609-665, 1994.

LUCCHESI, D. A realização do sujeito pronominal. In: LUCCHESI, D.; BAXTER, A.; RIBEIRO, I. (Orgs.) O Português Afro-Brasileiro. Salvador: EDUFBA, 2009. p. 167-183.

LUCCHESI, D.; BAXTER, A.; RIBEIRO, I. (Orgs.) O Português Afro-Brasileiro. Salvador: EDUFBA, 2009.

MACCLOSKEY, J.; HALE, K. On the syntax of person number inflection in Modern Irish. Natural Language and Linguistic Theory, v. 2, p. 487-533, 1984.

MAGALHÃES, T. M. V. As pessoas do discurso na produção de sujeitos nulos na aquisição do Português Brasileiro e do Português Europeu. ENCONTRO NACIONAL DE LÍNGUA FALADA E ESCRITA - ELFE, 5., Maceió, 2006. [handout].

MODESTO, M. Topic Prominence and Null Subjects. In: BIBERAUER, T. (ed.) The Limits of Syntactic Variation. Amsterdam: John Benjamins, 2008. v. 132 (Linguistics Today), p. 375-409.

MOURA, D. (Org.). Resquícios de Palmares: o que a comunidade quilombola nos diz. Maceió: EDUFAL, 2009.

NEELEMAN, A.; SZENDROI, K. Radical pro drop and the morphology of pronouns. Linguistic Inquiry, n. 38, p. 671-714, 2007.

NOYER, R. Features, positions and affixes in autonomous morphological structures. Tese (Doutorado) - MIT, Cambridge, 1992.

PREMINGER, O. Agreement and its Failures. Linguistic Inquiry Monograph, n.68. Cambridge, MA: MIT University Press, 2014.

RIBEIRO, I. O sujeito nulo referencial no português popular brasileiro - século XIX. In: TORRES MORAIS, M. A. C. R.; ANDRADE, L. C. V. O. (Orgs.). História do português paulista. Campinas, SP: UNICAMP/Publicações IEL, 2009. p. 83-98. 
. O sistema de definitude e de referencialidade de uma falante afro-brasileira idosa. ENCONTRO DA ABECS, 5., Salvador, 2010. [handout]

RIZZI, L. Null objects in Italian and the theory of pro. Linguistic Inquiry, v. 17, n.3, p. 501$557,1986$.

MIT University Press. 1990.

. Relativazed Minimality. Linguistic Inquiry Monograph, n.16. Cambridge:

ROBERTS, I. Creoles, markedness and the Language Bioprogram Hypothesis. Estudos Linguísticos e Literários, Salvador, n. 19, p. 11-24, 1997.

Ms. University of Cambridge, 2007.

. A deletion analysis of null subjects: French as a case study. Cambridge:

RICHARDS, M. Two Kinds of Variation in a Minimalist System. In: HECK, F.; MÜLLER, G.; TROMMER, J. (Eds.) Varieties of Competition. Leipzig: Linguistische Arbeits Berichte 87; Universität Leipzig, 2008.

SPEAS, M. Economy, agreement and the representation of null arguments. In: ACKEMA, P.; BRANDT, P.; SCHOORLEMMER, M.; WEERMAN, F. (Eds.) Agreement and argument structure. Oxford: OUP, 2004.

Submetido em: 12/02/2015

Aceito em: 27/04/2015 